\title{
ChemComm
}

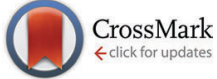

Cite this: Chem. Commun., 2016, 52, 1875

Received 10th November 2015, Accepted 4th December 2015

DOI: $10.1039 / \mathrm{c5cc09328g}$

www.rsc.org/chemcomm

\section{Versatile process for the stereodiverse construction of 1,3-polyols: iterative chain elongation with chiral building blocks $\dagger$}

\author{
Angela Bredenkamp, Michael Wegener, Sara Hummel, Andreas P. Häring and \\ Stefan F. Kirsch*
}

\begin{abstract}
A versatile process for the construction of 1,3-polyols, a key structural element of polyketide-type natural products, is presented. The modular synthesis strategy involves the iterative chain elongation with novel four-carbon building blocks to access all possible stereoisomers of a growing 1,3-polyol chain. These chiral building blocks are designed to install four carbon atoms with two stereogenic centres by performing only four experimentally simple steps per elongation cycle, thus making these building blocks attractive for the realization of a universal platform from which to access a diverse range of polyketidic molecules.
\end{abstract}

Polyketide-type natural products are a class of molecules with astonishing structural diversity. Their highly complex structures constitute a rich source of bioactive compounds, with many possessing therapeutic relevance as antibiotics and cardiovascular and antitumor agents, and thus are of particular interest from a pharmacological point of view. ${ }^{1}$ It is remarkable that the carbon skeletons underlying the vast range of polyketidic structures in Nature derive from a common biosynthetic origin where the controlled assembly is achieved with only a few simple building blocks (i.e., through repetitive $\mathrm{C} 2$-elongation of acetate- or propionate starter units by decarboxylative condensation with malonyl-CoA extender units). ${ }^{1 a-c}$ Despite an immense research endeavour to gain synthetic access to polyketide natural products and derivatives thereof, ${ }^{2}$ the construction of elaborate polyketidic structures remains highly challenging: the chemical total synthesis of polyketides involves, for example, the controlled formation of multiple stereocentres and might require somewhat sophisticated chemistry for realizing asymmetric carbon-carbon bond formations, resulting in syntheses that often assume a high level of complexity. ${ }^{3}$ However, to exploit the richness of biological activity offered by polyketidic structures more efficiently, convenient and general strategies for their construction have to be devised that ideally

Organic Chemistry, Bergische Universität Wuppertal, Gaußstr. 20,

42119 Wuppertal, Germany. E-mail: sfkirsch@uni-wuppertal.de

$\dagger$ Electronic supplementary information (ESI) available. See DOI: 10.1039/c5cc09328g proceed with a maximum of simplicity, which is of particular importance when considering the long-term goal of realizing such syntheses by fully controlled, automated processes. ${ }^{4,5}$

Motivated by the prospect of a synthetic access to polyketidic motifs that requires a minimum of planning and is preparatively easy to perform, we envisaged a highly modular strategy based on an iterative chain growth using chiral building blocks. We now wish to report the first stage of these efforts in the form of a convenient and efficient method for the stereocontrolled construction of 1,3 -polyols. ${ }^{6}$ Despite the fact that numerous synthetic methods for the stereoselective assembly of 1,3-diols were developed over the past decades, ${ }^{7}$ a number of iterative strategies have recently attracted renewed interest. ${ }^{8}$ In particular, strategies with catalytic asymmetric key steps have evolved in a highly useful way and with an impressive level of stereocontrol. ${ }^{9-11}$ However, the potential of chiral building blocks that allow the growth of polyol chains by iterative aldehyde olefinations was not recognized. ${ }^{12}$

We recently disclosed the application of chiral phosphine oxides 1 as building blocks for the stereospecific introduction of 1,3-diols in the synthesis of polyketidic structures; $(R)-\mathbf{1}$ and $(S)$-1 were accessible on a multigram scale through a facile and high-yielding sequence of six and seven linear steps, respectively. ${ }^{13}$ In this work, we expanded on that principle by using these four-carbon building blocks, which already carry preinstalled stereo-chemical information, in a repetitive chain elongation process as shown in Scheme 1. While, for example, Paterson et al. used boron-mediated aldol reactions for the synthesis of polyketide-type sequences, ${ }^{14}$ our strategy focused on the use of olefination reactions to attach the chiral building block $\mathbf{1}$ at the terminal aldehyde functionality $\mathbf{A}^{\mathbf{1}}-\mathbf{A}^{\boldsymbol{n}}$ in an iterative way. The attachment of the building blocks occurs without generating stereogenic centres by use of a carbonyl olefination followed by hydrolysis $(\rightarrow \mathbf{B}) \cdot{ }^{15,16}$ In consequence, by the selection of the chiral building block at each stage of the iterative sequence, the stereogenic centre that is part of the building block is unambiguously fixed. ${ }^{17}$ Regarding all the further reactions of the chain-extending sequence, we decided 

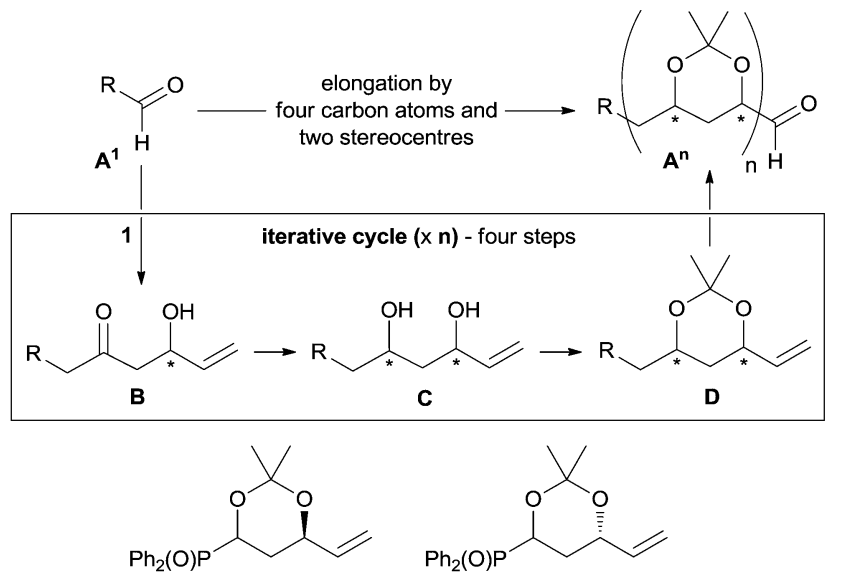

$(R)-1$

(S)-1

Scheme 1 General strategy for the synthesis of 1,3-polyols by use of four-carbon building block 1 .

only to rely on well-established and easy-to-perform reactions: substrate-controlled directed reduction of hydroxyl ketones $(\rightarrow \mathbf{C})$, hydroxyl protection $(\rightarrow \mathbf{D})$, and oxidative aldehyde formation.

The potential of this strategy to easily access any given 1,3-polyol chain in an enatioenriched manner was first put to the test starting from aldehyde 2 . As outlined in Scheme 2, the initial elongation started with $(R)-\mathbf{1}$ : by use of a short two-step sequence, the diols $\mathbf{4}$ and $\mathbf{5}$ were formed in high overall yields (77\% for $4 ; 76 \%$ for 5 ). In the course of this sequence, the aldehyde 2 was converted first into the olefination product through reaction with the lithiated building block $(R)-1{ }^{18}$ The resulting mixture of double bond isomers was not isolated;

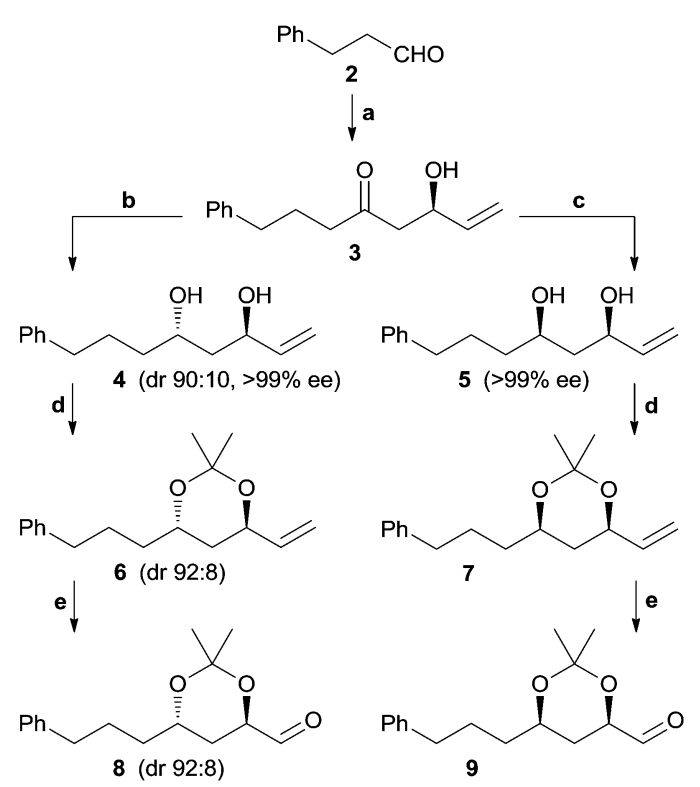

Scheme 2 Multistep sequence for the chain growth of 1,3-diols using building block 1 . Reagents and conditions: (a) (i) (R) $-\mathbf{1}, \mathrm{LDA},-78{ }^{\circ} \mathrm{C}, \mathrm{THF}$, (ii) $\mathrm{KOt}-\mathrm{Bu}$, r.t., (iii) $\mathrm{HCl}(1 \mathrm{~N}), 83 \%$; (b) $\mathrm{Me}_{4} \mathrm{NBH}(\mathrm{OAc})_{3},-35{ }^{\circ} \mathrm{C}$, MeCN/ $\mathrm{AcOH}, 93 \%$, dr $90: 10$; (c) $\mathrm{Et}_{2} \mathrm{BOMe}, \mathrm{NaBH}_{4},-78{ }^{\circ} \mathrm{C}, \mathrm{THF} / \mathrm{MeOH}, 91 \%$; (d) $\mathrm{Me}_{2} \mathrm{C}(\mathrm{OMe})_{2}, p-\mathrm{TSA}, 40{ }^{\circ} \mathrm{C}, 95 \%$, dr $92: 8$ for 6,92\% for 7; (e) (i) $\mathrm{O}_{3}$, $\mathrm{NaHCO}_{3},-78{ }^{\circ} \mathrm{C}, \mathrm{CH}_{2} \mathrm{Cl}_{2} / \mathrm{MeOH}$, (ii) $\mathrm{Me}_{2} \mathrm{~S}$, r.t., $88 \%$, dr $92: 8$ for $8,91 \%$ for 9. $\mathrm{LDA}=$ lithium diisopropylamide, $p$-TSA $=p$-toluenesulfonic acid. instead, the enol acetonide rapidly and quantitatively hydrolysed during the acidic work-up to provide ketone $3 .{ }^{19}$ Regarding the substrate-controlled reduction of the newly formed carbonyl group, we firstly relied on Evans' well-established method of directed anti-reduction to obtain the desired anti-diol 4 in 93\% yield (dr $90: 10) .^{20}$ Secondly, syn-diol 5 was accessed in $91 \%$ yield by use of $\mathrm{Et}_{2} \mathrm{BOMe} / \mathrm{NaBH}_{4} \cdot{ }^{21}$ The enantiomeric purity of the diols 4 and 5 was shown to be higher than $99 \%$ ee. Subsequent protection of the diols as acetonides followed by ozonolysis of the terminal olefin then gave the aldehydes 8 (anti) and $9(s y n)$ that represent the chain growth products of the iteration. $^{22,23}$ In brief, the overall chain elongation sequences with building block 1 comprise of only four synthetic steps and allow for the attachment of a four-carbon unit and the stereocontrolled installation of two stereogenic centres. ${ }^{24}$

To illustrate the ease of synthesising 1,3-polyols in this way, aldehydes 8 and $\mathbf{9}$ were subjected to further iterations of the four-step sequences to selectively prepare all eight possible diastereomers (10-17) of a tetraol skeleton in enantiopure form (Scheme 3). Although the average yields for the second iteration were, in particular when starting with anti-aldehyde 8, somewhat lowered (i.e., between $67 \%$ and $82 \%$ average yield per step), all target compounds were produced as expected, without any attempts to readjust experimental details or further optimise conditions.

Having thus demonstrated the stereochemical diversity of products accessible by this method, we next sought to apply it to the synthesis of a specific and more complex target. We decided on (protected) hexaol 19 as the desired product, which corresponds to the hexaol skeleton of (+)-cryptocaryol A (22) (Scheme 4). Due to its highly valuable biological properties (i.e. the stabilization of tumour suppressor protein Pdcd4), ${ }^{25,26}$ this natural product has attracted considerable interest, and its synthetic access has already been thoroughly investigated by several research groups. ${ }^{27-30}$ As a result, cryptocaryol A is analytically well documented, which would present us with the opportunity to confirm the structure and stereochemistry of desired hexaol 19 by further converting it into (+)-cryptocaryol A and comparing the NMR spectroscopic data with the literature. At the same time, its linear synthesis would underline the applicability of our method in natural product synthesis.

As detailed in Scheme 4, the iterative chain growth toward hexaol 19 using our method started from commercially available aldehyde 18. We were pleased to find that the synthesis via three iterations of the elongation cycle (the last of which was shortened by the ozonolysis step to stop at the terminal olefin) proceeded as smoothly and efficiently as hoped: a stereospecific elongation by thirteen carbon atoms and six stereogenic centres was achieved in eleven steps, with an average yield of $84 \%$ per step for all three iterations, to furnish hexaol 19 under full stereocontrol. Including the six-step synthesis of building block $(R)-1,{ }^{13}$ the longest linear sequence toward hexaol 19 consists of 17 steps. Regarding reaction conditions of the iteration steps, minor changes (specifically of solvents and reaction temperature) were required here in some instances due to solubility issues arising from the long aliphatic chain (see ESI $\dagger$ ). Lastly, to confirm structure and stereochemistry of the synthesised polyol chain 


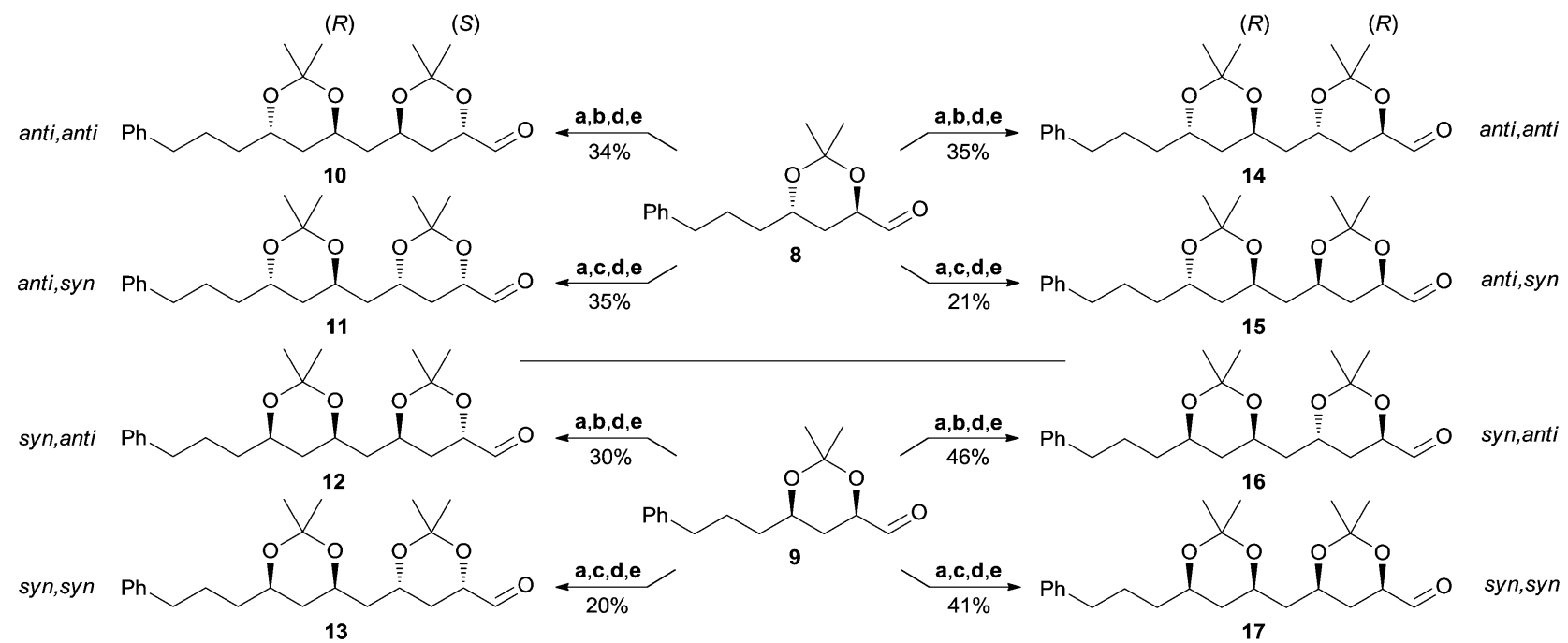

Scheme 3 Stereodiverse synthesis of tetraol-containing compounds. Reagents and conditions a-e as outlined in Scheme 1 using (R)- or (S)-building block 1 as appropriate (yields given for each iterative cycle over four steps; individual yields for each step a-e on the routes to 10-17 are detailed in the ESI $\dagger$ ).

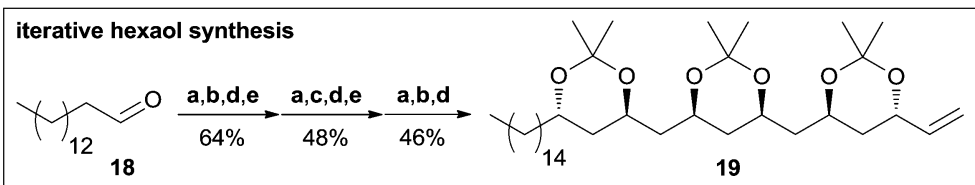

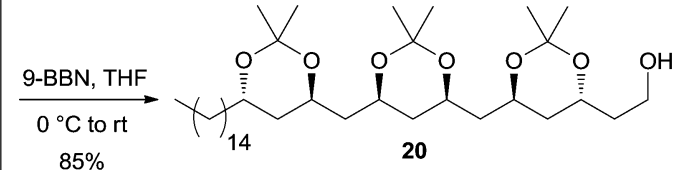

$85 \%$

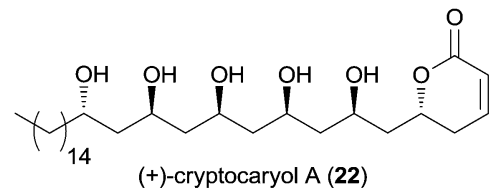

Scheme 4 Fully stereo-controlled synthesis of protected hexaol 19, with the target structure subsequently verified by further conversion into $(+)$-cryptocaryol A (22). Reagents and conditions a-e as outlined in Scheme 1 (with minor adjustments for certain steps, see ESI $\dagger$ ) using (R)- or (S)-building block 1 as appropriate (yields given for each iterative cycle over four, four, and three steps, respectively; individual yields for each step a-e on the route to 19 are detailed in the ESI†). 9-BBN = 9-borabicyclo[3.3.1]nonane, TPAP = tetrapropyl-ammonium perruthenate, $N M O=N$-methylmorpholine $N$-oxide, TFA $=$ trifluoroacetic acid, $p$-TSA $=p$-toluenesulfonic acid.

19, small amounts were subjected to a short series of manipulations to obtain a sample of (+)-cryptocaryol A: first, hydroboration of the olefin gave primary alcohol $\mathbf{2 0}$, which was then oxidised with TPAP followed by Ando olefination ${ }^{31}$ to yield conjugated ester 21. The natural product was accessed through universal deprotection and cyclization under acidic conditions; while the conversion of $\mathbf{2 1}$ with TFA effectively cleaved the acetonide protecting groups, further treatment with $p$-TSA was necessary to complete the final cyclization step. Thus, sufficient amounts of cryptocaryol A were obtained to positively match NMR spectroscopic data $\left({ }^{1} \mathrm{H},{ }^{13} \mathrm{C}\right)$ with those previously reported, ${ }^{25,28-30}$ which gave us verification that the desired hexaol 19 was indeed synthesised as planned. Comparison with the previous strategies for the synthesis of cryptocaryol A shows that our method with 22 linear steps (16 from aldehyde 18) is competitive regarding the step count (O'Doherty: 23, Cossy: 20, Dias: 17 linear steps for 22); ${ }^{28-30}$ however, our building block approach ensures full flexibility with respect to stereochemical variants of the target molecule.

In conclusion, a versatile process for the construction of 1,3-polyols, a key structural element of polyketide-type natural products, is described. Our modular synthesis strategy involves the iterative chain elongation with chiral four-carbon building blocks. All possible stereoisomers of a growing 1,3-polyol chain are accessible in a fully controlled manner, thus making these building blocks attractive for the realization of a universal platform from which to access a diverse range of polyketidic molecules. In our follow-up works, we will introduce several related building blocks that enable the incorporation of structural motifs other than pure 1,3-diols into synthetic polyketides.

The authors thank Dr Tobias Harschneck for seminal experiments in the field, and Phillip Biallas and Julian Vollrodt for further experimental support. The donation of chemicals by Rockwood Lithium is gratefully acknowledged.

\section{Notes and references}

1 (a) C. Hertweck, Angew. Chem., Int. Ed., 2009, 48, 4688; (b) Y.-H. Chooi and Y. J. Tang, Org. Chem., 2012, 77, 9933; (c) J. Staunton and K. J. Weissman, Nat. Prod. Rep., 2001, 18, 380.

2 For reviews, see: (a) A. K. Miller and D. Trauner, Synlett, 2006, 2295;

(b) B. Schetter and R. Mahrwald, Angew. Chem., Int. Ed., 2006, 45, 7506; (c) K.-S. Yeung and I. Paterson, Chem. Rev., 2005, 105, 4237. 
3 For recent examples, see inter alia: (a) T. J. Harrison, S. Ho and J. L. Leighton, J. Am. Chem. Soc., 2011, 133, 7308; (b) L. Wang and D. Menche, Angew. Chem., Int. Ed., 2012, 51, 9425; (c) K. C. Nicolaou, A. L. Nold, R. R. Milburn and C. Schindler, Angew. Chem., Int. Ed., 2006, 45, 6527.

4 For prime examples of efficient linear synthesis, see: (a) M. Burns, S. Essafi, J. R. Bame, S. P. Bull, M. P. Webster, S. Balieu, J. W. Dale, C. P. Butts, J. N. Harvey and V. K. Aggarwal, Nature, 2014, 513, 183; (b) S. Balieu, G. E. Hallett, M. Burns, T. Bootwicha, J. Studley and V. K. Aggarwal, J. Am. Chem. Soc., 2015, 137, 4398.

5 For an automated, iterative cross-coupling process, see: $(a) \mathrm{J}$. Li, S. G. Ballmer, E. P. Gillis, S. Fujii, M. J. Schmidt, A. M. E. Palazzolo, J. W. Lehmann, G. F. Morehouse and M. D. Burke, Science, 2015, 347,1221 . For a review on controlled iterative cross-coupling, see: (b) C. Wang and F. Glorius, Angew. Chem., Int. Ed., 2009, 48, 5240.

6 (a) S. D. Rychnovsky, Chem. Rev., 1995, 95, 2021; (b) S. E. Bode, M. Wolberg and M. Müller, Synthesis, 2006, 557; (c) C. Schneider, Angew. Chem., Int. Ed., 1998, 37, 1375; (d) T. Oishi and T. Nakata, Synthesis, 1990, 635.

7 For selected examples on substrate-controlled asymmetric induction, see: (a) J. W. Bode, N. Fraefel, D. Muri and E. M. Carreira, Angew. Chem., Int. Ed., 2001, 40, 2082; (b) D. A. Evans, K. T. Chapman and E. M. Carreira, J. Am. Chem. Soc., 1988, 110, 3560.

8 (a) H. Fuwa, S. Naito, T. Goto and M. Sasaki, Angew. Chem., Int. Ed., 2008, 47, 4737; (b) M. J. Mitton-Fry, A. J. Cullen and T. Sammakia, Angew. Chem., Int. Ed., 2007, 46, 1066; (c) J. D. Umarye, T. Leßmann, A. B. Garcia, V. Mamane, S. Sommer and H. Waldmann, Chem. - Eur. J., 2007, 13, 3305; (d) F. Allais, M.-C. Louvel and J. Cossy, Synlett, 2007, 451; (e) Z. Zhang, S. Aubry and Y. Kishi, Org. Lett., 2008, 10, 3077.

9 (a) Y. Lu, I. S. Kim, A. Hassan, D. J. Del Valle and M. J. Krische, Angew. Chem., Int. Ed., 2009, 48, 5018; (b) A.-M. R. Dechert-Schmitt, D. C. Schmitt and M. J. Krische, Angew. Chem., Int. Ed., 2013, 52, 3195; (c) S. B. Han, A. Hassan, I. S. Kim and M. J. Krische, J. Am. Chem. Soc., 2010, 132, 15559.

10 Methods based on asymmetric C-O bond formations: (a) J. T. Binder and S. F. Kirsch, Chem. Commun., 2007, 4164; (b) H. Menz and S. F. Kirsch, Org. Lett., 2009, 11, 5634; (c) S. F. Kirsch, P. Klahn and H. Menz, Synthesis, 2011, 3592; (d) K. C. Nicolaou, R. A. Daines, J. Uenishi, W. S. Li, D. P. Papahatjis and T. K. Chakraborty, J. Am. Chem. Soc., 1988, 110, 4672; (e) S. Tosaki, Y. Horiuchi, T. Nemoto, T. Ohshima and M. Shibasaki, Chem. - Eur. J., 2004, 10, 1527; $(f)$ N. B. Kondekar and P. Kumar, Org. Lett., 2009, 11, 2611; $(g)$ D. Kim, J. S. Lee, S. B. Kong and H. Han, Angew. Chem., Int. Ed., 2013, 52, 4203.

11 (a) B. J. Albert, Y. Yamaoka and H. Yamamoto, Angew. Chem., Int. Ed., 2011, 50, 2610; (b) B. J. Albert and H. Yamamoto, Angew. Chem., Int. Ed., 2010, 49, 2747; (c) M. Iwata, R. Yazaki, Y. Suzuki, N. Kumagai and M. Shibasaki, J. Am. Chem. Soc., 2009, 131, 18244; (d) P. B. Brady, S. Oda and H. Yamamoto, Org. Lett., 2014, 16, 3864.

12 In a series of works, 4-lithio-1,3-dioxanes were established as 1,3-diol synthons: (a) S. D. Rychnovsky and D. J. Skalitzky, Synlett, 1995, 555; (b) C. J. Sinz and S. D. Rychnovsky, Top. Curr. Chem., 2001, 216, 51; (c) N. A. Powell and S. D. Rychnovsky, J. Org. Chem., 1999, 64, 2026; (d) S. D. Rychnovsky and G. Griesgraber, J. Org. Chem., 1992, 57, 1559; (e) S. D. Rychnovsky and N. A. Powell, J. Org. Chem., 1997, 62, 6460; $(f)$ S. D. Rychnovsky, A. J. Buckmelter, V. H. Dahanukar and D. J. Skalitzky, J. Org. Chem., 1999, 64, 6849.

13 A. Bredenkamp, Z.-B. Zhu and S. F. Kirsch, Eur. J. Org. Chem., DOI: 10.1002/ejoc.201501325.
14 For a combinatorial approach to polyketides based on asymmetric aldol reactions, see: $(a)$ I. Paterson, M. Donghi and K. Gerlach, Angew. Chem., Int. Ed., 2000, 39, 3315; (b) I. Paterson and T. Temal-Laib, Org. Lett., 2002, 4, 2473.

15 For an iterative strategy toward 1,5-polyols based on chiral building blocks, see: G. K. Friestad and G. Sreenilayam, Org. Lett., 2010, $12,5016$.

16 For the use of chiral two-carbon building blocks, see: (a) J. R. Huckins, J. De Vicente and S. D. Rychnovsky, Org. Lett., 2007, 9, 4757; (b) R. Kartika, T. R. Gruffi and R. E. Taylor, Org. Lett., 2008, 10, 5047; (c) V. Malathong and S. D. Rychnovsky, Org. Lett., 2009, 11, 4220.

17 The plan was to keep the number of asymmetric transformations at a minimum to avoid problems arising from the chirality of the already established chain. For a review, see: S. Masamune, W. Choy, J. S. Peterson and L. S. Sita, Angew. Chem., Int. Ed. Engl., 1985, 24, 1.

18 (a) S. Ley, B. Lygo, H. M. Organ and A. Wannacott, Tetrahedron, 1985, 41, 3825; (b) J. I. Grayson and S. Warren, J. Chem. Soc., Perkin Trans. 1, 1977, 2263.

19 This smooth work-up protocol for removing the acetonide group led to a sum of four steps per iteration sequence (instead of five steps as expected at the start of the project). The acidic work-up proved highly selective for enol acetonides while other acetonides remained unaffected, thus allowing for the convenient protection of the growing 1,3-polyol chain as acetonides.

20 D. A. Evans, K. T. Chapman and E. M. Carreira, J. Am. Chem. Soc., 1988, 110, 3560.

21 (a) K. Narasaka and F. C. Pai, Tetrahedron, 1984, 40, 2233; (b) K.-M. Chen, G. E. Hardtman, K. Prasad, O. Repic and M. J. Shapiro, Tetrahedron Lett., 1987, 28, 155.

22 Though in some cases the reduction procedures led to diastereomeric mixtures, typically the minor diastereoisomer was removed in the course of the purification protocols connected with the acetonide formation and the ozonolysis.

23 The observed relative configuration of all the acetonides was assigned according to Rychnovsky's method: S. D. Rychnovsky, B. Rogers and G. Yang, J. Org. Chem., 1993, 58, 3511.

24 In comparison, iterative strategies based on asymmetric allylation typically require three synthetic steps per two-carbon elongation (i.e., six steps per four-carbon elongation, as done with building block 1 in four steps); see ref. 8.

25 For initial isolation and biological evaluation of cryptocaryol A, see: T. Grkovic, J. S. Blees, N. H. Colburn, T. Schmid, C. L. Thomas, C. J. Henrich, J. B. McMahon and K. R. Gustafson, J. Nat. Prod., 2011, 74, 1015.

26 (a) J. L. Cmarik, H. Min, G. Hegamyer, S. Zhan, M. Kulesz-Martin, H. Yoshinaga, S. Matsuhashi and N. H. Colburn, Proc. Natl. Acad. Sci. U. S. A., 1999, 96, 14037; (b) H. Yu, J. Zeng, X. Liang, W. Wang, Y. Zhou, Y. Sun, S. Liu, W. Li, C. Chen and J. Jia, PLoS One, 2014, 9, e105306; (c) A. P. Jansen, C. E. Camalier, C. Stark and N. H. Colburn, Mol. Cancer Ther., 2004, 3, 103.

27 For a synthesis of the originally reported structure of cryptocaryol A, see: D. S. Reddy and D. K. Mohapatra, Eur. J. Org. Chem., 2013, 1051.

28 For a structural revision of cryptocaryol A and the first total synthesis of the natural product, see: Y. Wang and G. A. O'Doherty, J. Am. Chem. Soc., 2013, 135, 9334.

29 E. Brun, V. Bellosta and J. Cossy, J. Org. Chem., 2015, 80, 8668.

30 L. C. Dias, P. K. Kuroishia and E. C. de Lucca Jr., Org. Biomol. Chem., 2015, 13, 3575.

31 K. Ando, J. Org. Chem., 1997, 62, 1934. 